\title{
Entropy Thresholding based Retinal Blood Vessel Segmentation
}

\author{
A. S. Deshpande ${ }^{1}$, Nayan A. Karale ${ }^{2}$ \\ ${ }^{1}$ Assistant Professor, Department of Electronics and Telecommunication, Savitribai Phule Pune University, Pune, Maharashtra, India \\ ${ }^{2}$ P.G. Student, Department of Electronics and Telecommunication, Savitribai Phule Pune University, Pune, Maharashtra, India
}

\begin{abstract}
Accurate segmentation of retinal blood vessel plays very important role for the efficient diagnosis of retinal vascular disorders. In this paper, Entropy based thresholding along with multiple features is proposed. Multiple features like vesselness measure, magnitude response of gaborfilter and gamma correction with inverted green channel are extracted. These extracted features are combined using neural network. Later, Entropy based thresholding is used to get the segmented blood vessel.
\end{abstract}

Keywords: Fundus image, Retinal Vascular Disorder, Segmentation, Entropy

\section{Introduction}

Optic fundus assessment has been widely used by the medical community for diagnosing vascular and nonvascular pathology. The retinal vasculature inspection may reveal hypertension, arteriosclerosis, diabetes, cardiovascular disease and stroke. The major cause of adult blindness is Diabetic retinopathy due to changes in blood vessel structure and distribution such as new vessel growth (proliferative diabetic retinopathy) and requires laborious analysis from a specialist. Endeavoring to reduce the effect of proliferative diabetic retinopathy includes obtaining and analyzing images of the optic fundus at regular intervals such as every six months to a year. Early recognition of changes to the blood vessel patterns can prevent major vision loss as early intervention becomes possible.

In order to provide the opportunity for initial assessment, computer based analysis has been introduced and to be carried out by community health workers which includes assessment of changes in the blood flow/vessel distribution due to either vessel narrowing, complete occlusions or new vessel growth and of the presence of microaneurysms. To carry out assessment of blood vessel proper segmentation of blood vessel plays very important role.

\section{Methodology}

The block diagram shown in fig. below is explained briefly which gives the flow for the Retinal blood vessel segmentation:

- Retinal Fundus Image: The Retinal Fundus Image is input image from DRIVE database.

- Preprocessing: Preprocessing is done to provide highest contrast for blood vessels by proving highest weight to green channel in YUV color space.

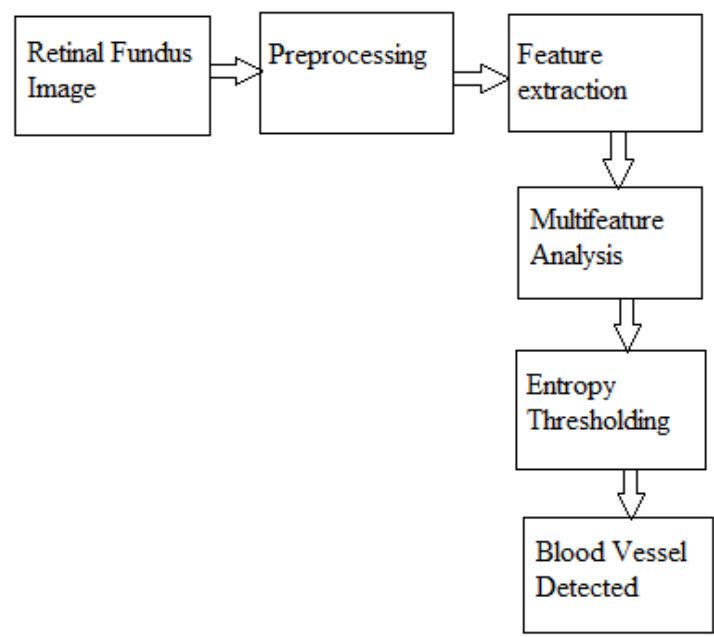

Figure 1: Block diagram of retinal blood vessel segmentation

- Feature extraction: Feature extracted are vesselness measure, response of gabor filter, gamma correction with inverted green channel.

- Multifeature Analysis: Multifeature Analysis is done by combing several features using multiperceptron neural network

- Entropy Thresholding: Entropy Thresholding is used to get segmented image with good accuracy.

\section{Entropy Thresholding}

This algorithm is based on Shannon's information entropy measure and is designed to maximize the amount of information between the two parts of a histogram as defined by a threshold point of histogram.

The process commences by first creating a histogram, partitioned into $\mathrm{n}$ parts, that ranges from the minimum to the maximum value of the extracted features. The concept of entropy based algorithm is on the separation of the histogram into two sections, $\mathrm{A}$ and $\mathrm{B}$, where the histogram of section $\mathrm{A}$ is from 1 to $s$ and the rest is in section $B$. The value associated with the histogram location of $\mathrm{s}$ is then considered as the threshold value.

$$
A: \frac{p_{1}}{P_{s}}, \frac{p_{2}}{P_{s}}, \ldots, \frac{p_{s}}{P_{s}}
$$




\section{International Journal of Science and Research (IJSR) \\ ISSN (Online): 2319-7064}

Index Copernicus Value (2013): 6.14 | Impact Factor (2014): 5.611

$$
\mathrm{B}: \frac{\mathrm{p}_{\mathrm{s}}+1}{1-\mathrm{P}_{\mathrm{s}}}, \frac{\mathrm{p}_{\mathrm{s}+2}}{1-\mathrm{P}_{\mathrm{s}}}, \ldots \ldots, \frac{\mathrm{p}_{\mathrm{n}}}{1-\mathrm{P}_{\mathrm{s}}}(2)
$$

where, the values $p_{i}: i=1, \ldots n$ are the probabilities associated with the partition in the histogram and $P_{s}=\sum_{i=1}^{n} p_{i}$.

The Shannon entropies associated with these sections A and $\mathrm{B}$ are computed by applying Shannon entropy equation for a single observation $\mathrm{X}$,

$$
\mathrm{H}(\mathrm{X})=\sum_{\mathrm{k}}^{\mathrm{n}}-\mathrm{P}_{\mathrm{k}} \log \mathrm{P}_{\mathrm{k}}
$$

The above equation is applied to section $\mathrm{A}$ and $\mathrm{B}$ in the histogram. These entropies are following,

$$
\begin{gathered}
\mathrm{H}(\mathrm{A})=-\sum_{\mathrm{i}=1}^{\mathrm{s}} \frac{\mathrm{p}_{\mathrm{i}}}{\mathrm{P}_{\mathrm{s}}} \log \frac{\mathrm{p}_{\mathrm{i}}}{\mathrm{P}_{\mathrm{s}}} \\
=\log P_{s}+\frac{H_{s}}{P_{S}}(4)
\end{gathered}
$$

And

$$
\begin{gathered}
H(B)=-\sum_{i=s+1}^{n} \frac{p_{i}}{1-P_{s}} \log \frac{p_{i}}{1-P_{s}} \\
=\log \left(1-P_{S}\right)+\frac{H_{n}-H_{s}}{1-P_{s}}
\end{gathered}
$$

Where $H_{n}=-\sum_{i=1}^{n} p_{i} \log p_{i}$ and is defined as the entropy over the whole histogram, and $H_{s}=-\sum_{i=1}^{s} p_{i} \log p_{i}$ and is the entropy of the section in the histogram from the start to the threshold location s.

Defining $\Psi(s)$ as the sum of $\mathrm{H}(\mathrm{A})$ and $\mathrm{H}(\mathrm{B})$,

$$
\Psi(s)=\log P_{s}\left(1-P_{s}\right)+\frac{H_{s}}{P_{s}}+\frac{H_{n}-H_{s}}{1-P_{s}}
$$

The threshold value is then defined as the location in the histogram where $\Psi(s)$ is maximum. This threshold value is further used for segmentation of retinal blood vessels.

\section{Algorithm}

1) Input Retinal Fundus image

2) Preprocessing of Retinal Fundus image

3) Feature extraction:

i) Vesselness Measure by Frangi

ii)Vesselness Measure by Salem

iii)Multiscale Gabor filter

iv) Gamma corrected using inverted green channel

4) Multifeature Analysis by combining all extracted feature

5) Entropy thresholding for blood vessel detection.

7) Retinal Blood Vessels detected.

\section{Results}

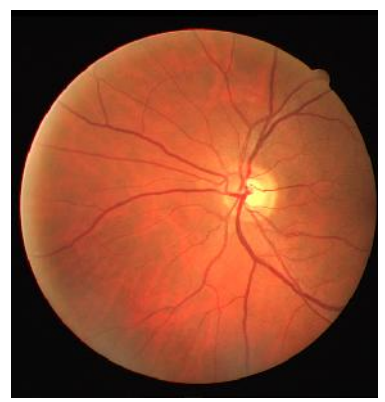

(a)

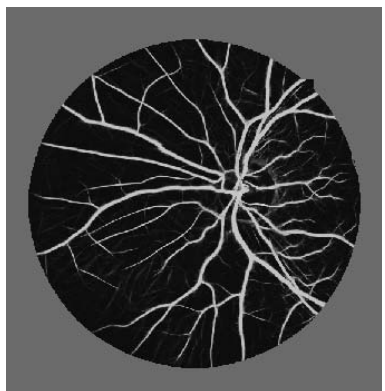

(c)

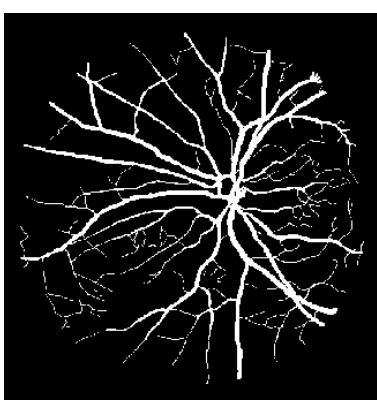

(b)

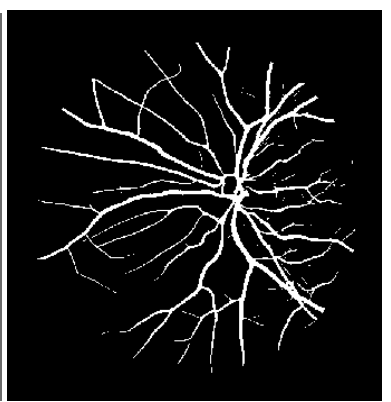

(d)

Figure 2: (a) Input Retinal Fundus Image, (b)Groundtruth Image, (c) Multifeature Analysis output, (d) Entropy Thresholding output

The input image is Retinal Fundus image from DRIVE Database. Here each image has been JPEG compressed.

Multifeature Analysis is done by combining several features using multiperceptron neural network. The several features are Vesselness measures, Magnitude response of Gabor filter and inverted green channel component. Fig. shows output of multifeature analysis.

The output of multifeature analysis is gray scale confidence image generated by neural network which is further thresholded using entropy thresholding. Entropy thresholding is designed to maximize the amount of information between the two parts of histogram as defined by threshold point of histogram. The entropy thresholding results in the segmentation of blood vessel which is shown in figure 2

The performance of proposed method is evaluated in terms of accuracy (Acc.), sensitivity (Se), specificity(Sp) and area under curve $\left(A_{Z}\right)$.

Table 1: Results of proposed method

\begin{tabular}{|l|l|l|l|}
\hline Images & Accuracy & Sensitivity & Specificity \\
\hline Img.1 & 0.961607 & 0.709341 & 0.98632 \\
\hline Img.2 & 0.957210 & 0.629772 & 0.994567 \\
\hline Img.3 & 0.951394 & 0.626455 & 0.987373 \\
\hline Img.4 & 0.961614 & 0.667886 & 0.991372 \\
\hline Img.5 & 0.957889 & 0.614939 & 0.993339 \\
\hline Img.6 & 0.952503 & 0.553618 & 0.995514 \\
\hline Img.7 & 0.957428 & 0.597207 & 0.993656 \\
\hline Img.8 & 0.951915 & 0.533693 & 0.991286 \\
\hline Img.9 & 0.955558 & 0.477880 & 0.997685 \\
\hline Img.10 & 0.962683 & 0.654404 & 0.99033 \\
\hline Img.11 & 0.956137 & 0.615322 & 0.989648 \\
\hline
\end{tabular}

\section{Volume 5 Issue 1, January 2016}


International Journal of Science and Research (IJSR)

ISSN (Online): 2319-7064

Index Copernicus Value (2013): 6.14 | Impact Factor (2014): 5.611

\begin{tabular}{|l|l|l|l|}
\hline Img.12 & 0.961759 & 0.658336 & 0.990434 \\
\hline Img.13 & 0.952485 & 0.572615 & 0.993648 \\
\hline Img.14 & 0.961862 & 0.685347 & 0.986185 \\
\hline Img.15 & 0.955498 & 0.707250 & 0.974633 \\
\hline Img.16 & 0.960001 & 0.613172 & 0.994423 \\
\hline Img.17 & 0.957125 & 0.530267 & 0.996478 \\
\hline Img.18 & 0.964926 & 0.642212 & 0.992696 \\
\hline Img.19 & 0.971703 & 0.801249 & 0.987121 \\
\hline Img.20 & 0.965208 & 0.666433 & 0.988924 \\
\hline Avg. & $\mathbf{0 . 9 5 8 8 3 4 2}$ & $\mathbf{0 . 6 2 7 8 6 9 9}$ & $\mathbf{0 . 9 9 0 7 8 1 6}$ \\
\hline
\end{tabular}

References

[1] Chaudhuri S, Chatterjee S, Katz N, Nelson M, and Goldbaum M, "Detection of blood vessels in retinal images using two-dimensional matched filters," IEEE Transactions on Medical Imaging, vol. 8, no. 3,pp. 263-269, 1989.

[2] Staal J, Abramoff MD, Niemeijer M, Viergever MA, and van Ginneken B, "Ridge-based vessel segmentation in color images of the retina,"IEEE Transactions on Medical Imaging, vol. 23, no. 4, pp. 501-509,2004.

The above table shows the results for entropy thresholding applied on the output of the multifeature analysis. It can be seen that specificity and accuracy is increased and sensitivity is comparatively less. This is because true negative pixels (correctly classified as non vessel) are comparatively more than true positive (correctly classified as vessel pixel).

Table 2: Comparison of Entropy thresholding with Fixed thresholding

\begin{tabular}{|l|l|l|l|}
\hline \multicolumn{1}{|c|}{ Methods } & \multicolumn{1}{c|}{ Accuracy } & Sensitivity & Specificity \\
\hline Fixed thresholding & 0.903685 & 0.894735 & 0.905094 \\
\hline Entropy thresholding & 0.95883425 & 0.6278699 & 0.9907816 \\
\hline
\end{tabular}

From the above table, overall accuracy of retinal blood vessel segmentation using entropy thresholding is more compared to that of fixed thresholding though sensitivity is less comparatively.

Table 3: Comparison of proposed method with other existing methods

\begin{tabular}{|c|l|c|c|c|}
\hline $\begin{array}{c}\text { Sr. } \\
n o\end{array}$ & \multicolumn{1}{|c|}{ Methods } & Accuracy & Sensitivity & Specificity \\
\hline 1 & $\begin{array}{l}\text { Emary et.al } \\
{[14]}\end{array}$ & 0.939 & 0.721 & 0.971 \\
\hline 2 & Fraz et. al.[13] & 0.9476 & 0.7525 & 0.9722 \\
\hline 3 & $\begin{array}{l}\text { Dhara et. } \\
\text { al.[12] } \\
\text { (Using fixed } \\
\text { thresholding) }\end{array}$ & 0.885 & 0.906 & 0.845 \\
\hline 4 & $\begin{array}{l}\text { Dhara et. } \\
\text { al.[12] } \\
\text { (Using } \\
\text { adaptive } \\
\text { thresholding) }\end{array}$ & 0.900 & 0.919 & 0.855 \\
\hline $\mathbf{5}$ & $\begin{array}{l}\text { Proposed } \\
\text { method }\end{array}$ & $\mathbf{0 . 9 5 8 8 3 4 2}$ & $\mathbf{0 . 6 2 7 8 6 9 9}$ & $\mathbf{0 . 9 9 0 7 8 1 6}$ \\
\hline
\end{tabular}

\section{Conclusion}

In many applications of ophthalmology, the most important step is to detect the blood vessels in the retina for diagnosing various vascular diseases. For proper diagnosis of the diseases, it is important to segment blood vessels more accurately.

Thus, multifeature analysis along with entropy thresholding is proposed to improve accuracy and specificity of retinal blood vessel segmentation. We have obtained the various feature like vesselness measure, gabor filter and gamma correction on inverted green channel. Later, Multifeature analysis is done by combining these several feature using neural network for obtaining confidence image. Then entropy thresholding is used to get the segmented blood vessel.

[3] "DRIVE: Digital Retinal Images for Vessel Extraction," www. isi.uu.nl/Research/ Databases/ DRIVE/ download.php, last accessed on June 21,2011.

[4] Soares JVB, Leandro JJG, Cesar Jr. RM, Jelinek HF, and Cree MJ, "Retinal vessel segmentation using the 2-D Gabor wavelet and supervised classification," IEEE Transactions on Medical Imaging, vol. 25,no. 9, pp. 1214-1222, 2006.

[5] Frangi AF, Niessen WJ, Vincken KL, and Viergever MA, "Multiscalevessel enhancement filtering," in Medical Image Computing andComputer-Assisted Intervention (MICCAI'98), vol. 1496 of LectureNotes in Computer Science, pp. 130-137. Springer, Berlin, Germany, October 1998.

[6] Salem MN, Salem AS, and Nandi AK, "Segmentation of retinal blood vessels based on analysis of the Hessian matrix and clustering algorithm," in 15th European Signal Processing Conference (EUSIPCO2007), Poznan, Poland, September 2007, pp. 428-432.

[7] Rangayyan RM, Ayres FJ, Oloumi Faraz, Oloumi Foad, and Eshghzadeh-Zanjani P, "Detection of blood vessels in the retina with multiscale Gabor filters," Journal of Electronic Imaging, vol. 17, pp. 023018:1-7, April-June 2008.

[8] Gonzalez RC and Woods RE, Digital Image Processing, Prentice Hall,Upper Saddle River, NJ, 2nd edition, 2002

[9] Rangayyan RM, Zhu X, Ayres FJ, and Ells AL, "Detection of the optic nerve head in fundus images of the retina with Gabor filters and phase portrait analysis," Journal of Digital Imaging, vol. 23, no. 4, pp.438-453, August 2010.

[10] M.M. Fraza, P. Remagninoa, A. Hoppea, B. Uyyanonvarab, A.R. Rudnickac,C.G. Owenc, S.A. Barmana ,Blood vessel segmentation methodologies in retinal images- A survey," Computer Methods And Programs In Biomedicine I08 (2012)407-433.

[11] Zhang B, Zhang L, Zhang L, Karray F,"Retinal vessel extraction by matched filter with first-order derivative of Gaussian,"Comput Biol Med. 2010 Apr;40(4):438-45.

[12] A.K. Dhara, R. M. Rangayyan, F. Oloumi, S. Mukhopadhyay, "Methods for thedetection of blood vessels in retinal fundus images and reduction of falsepositivepixels around the optic nerve head," in Proc. 4th IEEE International Conference on E-Health and Bioengineering - EHB 2013, Iasi, Romania, November 2013,pp. 1-6.

[13] M.M.Fraz,S.A.Barman,P.Remagnino,A.Hoppe,A.Basit, Uvyanonvara,A.R.Rudnicka, and CG Owen, "An approach to localize the retinal blood vessels using bit planes and centerline detection",Comput. Methods Programs Biomed.,2011.

[14] E.Emary, H.M.Zawbaa, A.E.Hassanien, G.Schaefer,A.T. Azar,"Retinal Blood Vessel Segmentation using Bee Colony Optimisation and Pattern Search,'International Joint Conference on Neural Network(IJCNN),Beijing ,China,July6-11,2014 